\title{
MILP of multitask scheduling of geographically distributed maintenance tasks
}

\author{
Hamed Allaham ${ }^{\mathrm{a}^{*}}$ and Doraid Dalalah ${ }^{\mathrm{a}, \mathrm{b}}$
}

${ }^{a}$ Industrial Engineering and Engineering Management, University of Sharjah, United Arab Emirates ${ }^{b}$ Industrial Engineering - Jordan University of Science and Technology, Jordan

\section{CH R O I C L E ABSTRACT}

\begin{tabular}{l} 
Article history: \\
Received June 12021 \\
Received in Revised Format \\
June 122021 \\
Accepted July 12021 \\
Available online \\
July, 1 2021 \\
\hline Keywords: \\
Maintenance \\
Scheduling \\
Routing \\
Task Assignment \\
Utilization
\end{tabular}

\section{Introduction}

Maintenance can be defined as a combination of both technical and administrative activities that are necessary to maintain or restore an entity or a system back to its working condition (British Standards Institution, 1984). However, maintenance priorities are not alike, which can be outlined in four targeted priority headings: ensuring system operation; ensuring system existence; ensuring safety; and ensuring human well-being. In nearly all fields of production and services, maintenance remains an important part for business survival (Dekker, 1996). To increase the performance of any system, the reliability of its components must be enhanced to decrease breakdowns and failures. The system's reliability can be improved by undertaking preventive maintenance according to schedules that consider various factors, such as the lifespan of the assets, available services, replacement parts, expenses, functional experience, etc. However, given the minimal intermission break time and the insufficient repair facilities along with limited budgets, it is often difficult to conduct top-level maintenance operations on all components of a system. Therefore, it becomes important to selectively choose the components that could have a greater effect on a system's reliability. The choice of which tasks to perform in a maintenance schedule leads to the so-called Selective Maintenance (SM). SM has been extensively analyzed in many research studies that enriched the literature with a good deal of interesting models (Cho \& Parlar, 1991).

\footnotetext{
* Corresponding author

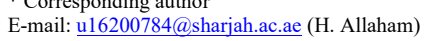

2022 Growing Science Ltd. doi: $10.5267 /$ j.ijiec.2021.7.001
} 
Valuable enhancements and extensions have been made in the selective maintenance literature following the early work of Rice et al. (1998). A good survey about maintenance styles and its optimization models can be found in Patwardhan et al. (2016). Maintenance service providers need to seek better maintenance models and better use of resources to reduce the costs and accomplish the necessary service agreement and achieve quality maintenance. Maintenance is generally divided into the three categories (Dhillon, 2002):

- Proactive maintenance: In this category, all operations are carried out in the testing and reconditioning phase on a planned, frequent, and reliable basis to maintain an item/equipment in a specified working condition.

- Corrective maintenance: In this category, unplanned maintenance or repair to return items/equipment to a certain state, carried out because maintenance persons or consumers observed unexpected flaws or faults.

- Predictive maintenance: It is a modern approach utilizing real-time feedback and signal processing methods to correctly forecast and diagnose the items/equipment status during service.

Today, technology is one of the core pillars used to track and optimize maintenance schedules, workforce, maintenance hours, and work performance to sustain the maintenance requirements. The working conditions of the assets can be observed and endured with high-tech real-time job monitoring systems. This is achieved by delivering timely maintenance considering the cost, lifespan of parts, system performance, and maintenance frequencies. Effective tracking of system status along with timely measures would improve systems stability and systems lifetime while reducing the maintenance costs simultaneously. High tech solutions have proven their reliability particularly in geographically distributed infrastructures. In systems of spatially distributed components, the travel between maintenance sites for either testing, preventive or corrective maintenance makes the maintenance more stressful. It was reported in many literature studies that travelling time between maintenance sites consumes a proportion of the available maintenance time (Camci, 2015; Rashidnejad et al., 2018). For this reason, a model that can put optimized maintenance schedules into operation while taking into consideration the burden of distant sites and travel time would significantly help improve the maintenance operations and increase the time utilization.

To address the above special category of maintenance operations, this paper will focus on maintenance activities in geographically scattered infrastructures. This form of service can be found in the maintenance contracts of commercial buildings, which include electrical, mechanical, plumbing, civil and advanced system maintenance activities such as fire alarms, firefighting, protection services, vertical transport systems, and other maintenance tasks. In this category, to arrive at the maintenance sites, the scattered locations of the small tasks require travelling at comparatively low speeds. To the best of our knowledge, limited work has been carried out to address preventive maintenance activities of spatial distribution and multi-skilled teams using MILP of exact solutions. Therefore, in this work, an original optimization model is introduced which considers the following cost components: staff, service level, spare parts as well as repair persons' travel cost subject to realistic schedule, time and capacity constraints. The goal of the model is to present maintenance schedules that take into consideration scarce resources such as the available time, the available technicians and the travel time between task sites. To validate the proposed model, different scenarios and case studies are considered from one of the renowned places that incorporate third-party maintenance providers to serve commercial buildings in the United Arab Emirates. Due to the huge business growth and the country's booming commercial sector and the rising number of residential ventures, maintenance service providers continuously seek better management that can efficiently address the different challenges in maintenance operations and contracts which make the selected case studies a good fit for our model. UAE facilities management industry stood at $\$ 13.8$ billion and is expected to rise to $\$ 27$ billion by 2024 at a compound annual growth rate of over 10 percent (www.globenewswire.com). The two major cities in the country, Dubai and Abu Dhabi, are experiencing dramatic growth in the real estate market, which is further increasing the demand for facility management across the country. Besides, the technological advancement in facilities, services, and management in the country along with accommodating World Expo 2021 will further boost the growth of the UAE facilities management industry over the next five years (research and markets, 2019). In general, most of the commercial buildings in UAE target very high maintenance standards to improve the quality of services offered to customers. The owners of commercial buildings seek more leaseholders, which cannot be attained except by offering reliable assets to stay in line with the international standards like ISO 9001.

Given the original proposed model of this work and the selected case studies, our study should contribute to the literature by addressing multi tasks, multi team-task assignments under capacity, distance, travel time and scheduling constraints. The model solution recommends collaborative decisions that include the number of maintenance teams, routes, tasks schedules, all detailed to days and teams. The model should concurrently reduce the cost of labor, replacement parts, penalties on service levels, and travel times.

\section{Literature Review}

The maintenance of commercial buildings, which is characterized by its distributed maintenance sites, is especially important for customers. While the scope of a typical plant/manufacturing maintenance may not be in direct contact with customers, the case is different in commercial buildings where the line of visibility of failures and breakdowns is close to the customers. Therefore, preventive and prompt maintenance actions need to be scheduled for fast response and recovery. While different 
approaches exist to address such maintenance requirements, optimization has been proven to be one of the efficient tools that demonstrate reliable solutions. However, few recent studies can be found on the area of repairman travelling problem.

Among few studies, a multi-period technician routing and scheduling problem was discussed by Zamorano and Stolletz (2017) to decide the regular distribution of technicians to teams, teams to duties, and teams to daily routes such that the cost of service is reduced. A branch-and-price algorithm is used to solve their model. In another more recent research, a genetic algorithm to minimize the present value of the costs and maximize the life cycle of the track-bed under different reliability levels and probabilistic degradation models was addressed by Sara Bressi et al. (2021). Earlier studies addressing preventive maintenance operations in different infrastructures can be found such as the model presented by Yu and Seif (2016) who explored a mixedinteger linear model integrating scalable and complex maintenance operations to reduce the overall tardiness and maintenance costs in a permutation flow shop scheduling. Distributed aging networks were addressed by Doostparast et al. (2014) who have discussed the preventive maintenance of coherent networks of aging elements.

One common aspect that was not satisfactorily taken into account in many maintenance studies including the above, is the required time to travel between the assets' sites and thus most of this work is still behind from the point of view of geographically scattered infrastructures (e.g. power supply, transport, telecommunications, utilities, etc.). Among the few studies that touched the geographical aspect of task sites, a bi-objective model for preventive maintenance considering predictive knowledge and residual useful life in geographically distributed ATMs was addressed by Rashidnejad, et al., (2018). To solve this difficult NP problem, a multi-objective metaheuristic method called Non-Dominant Sorting Genetic Algorithm II (NSGA-II) was built. A close study to lead the maintenance and scheduling of offshore wind turbines was presented by Fan et al., (2019), where a hybrid heuristic optimization strategy has been suggested for the solution. In their work, an integrated mixed particle swarm optimization (MPSO) and a discrete wolf pack search (DWPS) were employed to minimize the overall cost, including travel expenses, technicians, and penalties.

Another recent study can be found in Hedjazi et al. (2019) who proposed a new scheduling approach that considers maintenance operations underperformance restrictions for geographically scattered infrastructure. The suggested solution has the potential to simplify agreements between agents for repair operations and agents for human resources. Considering a fuzzy multi-objective non-linear chance-constrained programming model, the scheduling of preventive maintenance problems of wind farms in offshore wind energy fields was analyzed by Zhong et al., (2019).

The problem of planning and scheduling the maintenance of sewerage services for a variety of geographically dispersed locations subject to unexpected deficiencies and insufficient crews has been addressed by Fontecha et al., (2020). In their work, the path for a set of crews to conduct the scheduled maintenance activities at a near-minimum estimated cost per unit time was considered by linear programming. In the same context but reliability focused, a study was done by Yang et al., (2019) who discussed a scheduled planned maintenance problem of a two-component system that is evenly spaced. The aim was to jointly optimize the maintenance interval and the number of maintenance windows using the algorithm of the artificial bee colony.

For the routing of a single technician though, good yet limited research results can be found. A mixed-integer linear programming model was suggested for a multi-attribute routing and scheduling problem of a single technician performing maintenance of electronic transaction equipment was discussed by Mathlouthi, et al., (2018). In their settings, the technicians' route to execute activities at various customer locations is addressed for the purpose of increasing the gross benefit associated with customers served. A new reliable maintenance work scheduling to simultaneously reduce the total planned maintenance costs and optimize the overall tasks durations under unpredictable circumstances was addressed by the tasks (Golpîra \& Tirkolaee, 2019). In their research, the optimization was modeled via Bi-Objective Mixed-Integer Linear Programming (BOMILP) model. The findings further illustrated the supremacy of the suggested system in the scheduling of maintenance operations, taking into account their nominal frequencies.

A few studies have sought to merge the mechanism of employee routing with scheduling for repairs. Camci (2015) and Irawan et al., (2017) accomplished this for geographically scattered assets assuming that only one maintenance team is available, and each asset can be handled only once during the planning horizon. In a similar work, Chen et al. (2017) studied the integrated optimization of maintenance scheduling and staff routing for a drainage system with general network topology. Their goal was to minimize the flood risk of the whole system.

What we can conclude to the best of our knowledge, is that none of the above studies addresses multi tasks, multi team-task assignment under capacity, distance, travel time and topology constraints. In fact, real-world infrastructures of a general network topology usually have more complex settings than those linked to the component-level efficiencies considered in these studies. For this reason, and to find the right balance between maintenance expenses and system benefits in plants or facilities, we propose a cost Mixed Integer Linear Programming Model that considers the above settings. The model solution recommends collaborative decisions that include the number of maintenance teams, routes, tasks schedules, all detailed to days and teams. The model will reduce the cost of labor, replacement parts, penalties on service levels, and travel times. 


\section{Maintenance Scheduling vs. Maintenance Routing}

In maintenance operations of systems of geographically distributed infrastructures like commercial buildings, traveling between the task sites consumes a good deal of time. This time varies between the tasks as per exact tasks' locations, routes, and physical movement of each maintenance team. To reduce the time lost in moving between the maintenance sites, shortening the daily maintenance routes become essential particularly when the travel speed gets low.

The travel time of technicians between tasks in the maintenance of commercial buildings consumes a significant proportion of the available daytime. In some cases, maintenance teams may have to walk or use a slow electrical shuttle exploring their ways between pedestrians or shoppers in big malls to reach their maintenance sites (Suen \& Sen, 2004). A team performing a set of maintenance tasks every day has to decide on the best routes to follow across the tasks' sites, which, if not optimized, will affect the availability of time and travel costs. A routing problem results for each team every day when the distances between the task locations are identified. Fig. 1 shows the notion of two teams performing maintenance tasks at different sites over two days. Note that each team travels on a daily basis between selected tasks. The choice of tasks' sequence will affect the travel distance and therefore, the travel time between buildings. This means that every day, a travel salesman (TSP-like) problem will result for each team, which will make the problem expressively difficult to model and solve. Not only that, it is not exactly the same dilemma that is found in the classical TSP, this because a task that can be done today may be delayed to another day to avoid long travel, or may be assigned to another maintenance team, not to mention the time and technician availability constraints.

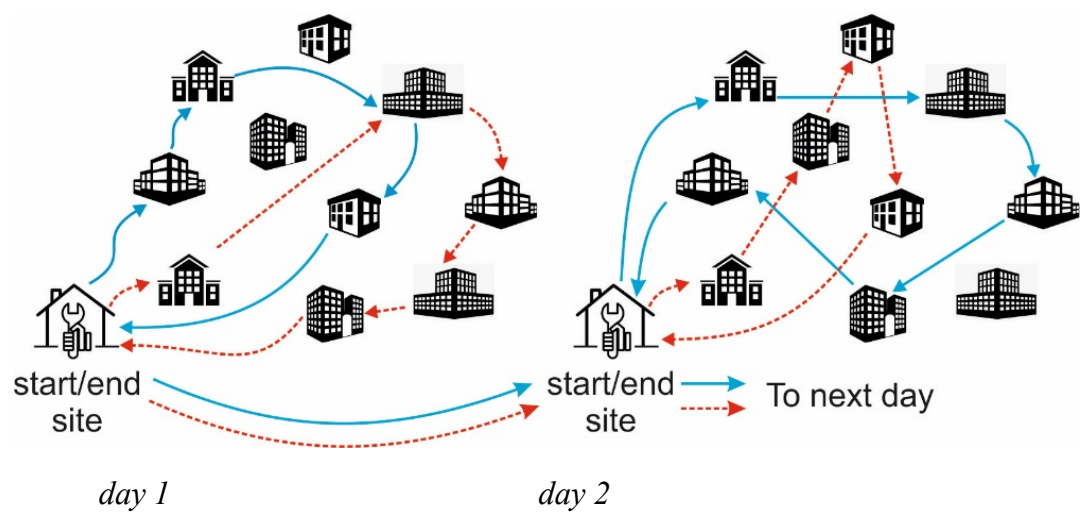

Fig. 1. Suggested maintenance routes of two maintenance teams over two days

\section{The Model}

A maintenance optimization model is formulated in this section to perform the following: optimize various cost elements, determine the best maintenance routes, assign the minimum number of work teams, and coordinate the activities over a predefined scheduling period. Here, a series of spatially distributed maintenance tasks $\{1, \ldots, M\}$ are to be performed by a subset of available maintenance teams $\{1, \ldots, W\}$ subject to some frequency in a $D$-long planning period, where $D$ is given in days. The model aims at providing a feasible time schedule and sequence of maintenance activities for geographically distributed task sites such as those encountered in commercial buildings by using a minimum number of maintenance teams. The objective function consists of four cost components subject to various time and capacity constraints, specifically, the cost of the hired teams, the cost of non-compliance with the required task frequencies (service level cost), the cost of carrying out additional tasks, and the cost of moving between task sites.

To establish such an objective function, a binary matrix $z$ is introduced which consists of the elements $z_{m w d}, \forall m=1, \ldots, M$, $\forall w=1, \ldots, W, \forall d=1, \ldots, D$, where $z_{m w d}=1$ if a maintenance task $m$ is performed by a team $w$ on day $d$, and $z_{m w d}=0$ otherwise. In this model, we assume multi-skill teams in which any team can perform any task, however, a task cannot be performed more than once a day, which can be modeled by the inequality $\sum_{w=1}^{W} z_{m w d} \leq 1$. Under the notation of the matrix $z$, the summation $u_{m d}=\sum_{w=1}^{W} Z_{m w d}$ will yield a two-dimensional binary matrix that represents the daily schedule of all tasks, where $u_{m d}=1$ if task $m$ is performed in day $d$ and " 0 " otherwise.

Although we start with $W$ teams, however, not all have to be hired, therefore, the variable $h_{w}$ will denote the hiring status where:

$$
h_{w}=\left\{\begin{array}{rr}
1 & \text { if team } w \text { is hired } \\
0 & \text { otherwise }
\end{array}\right.
$$

To this end, we can easily show that the condition $z_{m w d} \leq h_{w}$ must hold for $\forall m=1, \ldots, M, \forall w=1, \ldots, W$ and $\forall d=$ $1, \ldots, D$. Now, since part of our intention is to find the routes traversed by each maintenance team which should be spatially minimized to avoid extra traveling time between the maintenance sites, a variable $x_{w d i j}$ is introduced, where $x_{w d i j}=1$ if 
team $w$ moves from a site $i$ to a site $j$ in day $d$ and " 0 " otherwise. With this in mind, any team $w$ that is not hired results in a " 0 " value of the variable $x_{w \bullet \bullet}$, otherwise the value of $x_{w \bullet \bullet \bullet}$ is either 0 or 1 , ("•" refers to all values of the associated index) this is: $x_{w d i j} \leq h_{w}, \forall i, j=1, \ldots, M, \forall w=1, \ldots, W, \forall d=1, \ldots, D$. Not only the variable $x_{w d i j}$, the summation of the variable over $i$ or $j$ also indicates that only one site can be visited from/after $i$ or $j$ if team $w$ is hired. These conditions can be modeled by:

$$
x_{w d i j} \leq h_{w}, \sum_{j=1}^{M} x_{w d i j} \leq h_{w} \text { and } \sum_{i=1}^{M} x_{w d i j} \leq h_{w}, \forall m, \forall w, \forall d .
$$

There is a nominal frequency required by each task, meaning that each task must be performed a specific number of times in the maintenance planning period. A task that is short in the number of times it was performed results in a low service level and thus a shortage cost. Clearly, the number of times a task $m$ is performed in the planning period is equivalent to $\sum_{d=1}^{D} u_{m d}$ and the number of tasks that are performed in a specific day $d$ is given by $\sum_{m=1}^{M} u_{m d}$. A shortage is observed if $\sum_{m=1}^{M} u_{m d}<$ $f_{m}$ where $f_{m}$ is the nominal frequency of task $m$.

Likewise, a task $m$ is performed more than the required frequency if $\sum_{m=1}^{M} u_{m d}<f_{m}$. By assumption, a task cannot be performed more than the maximum number of days in the planning period. The percentage of shortage $s h_{m}$ can be found using the expression in Eq. (3):

$$
s h_{m}=\frac{f_{m}-\sum_{d=1}^{D} u_{m d}}{f_{m}} .
$$

While short tasks are expressed in terms of the percentage and thus entailing penalties on service levels as it is usually the case in commercial contracts, for the extra tasks, only the count of tasks is considered. The count of extra tasks, if it happens, can be given by the following expression:

$$
e_{m}=\sum_{d=1}^{D} u_{m d}-f_{m}
$$

Let the cost of hiring a team $w$ be $C_{w}$ per hr. The total cost of hired teams is thus given by $\sum_{w}^{W} h_{w} C_{w} D \times l$, where $l$ is the number of working hours per day. Under such notation, our model should minimize the following subject to the subsequent constraints:

$$
\min \left(\frac{R}{s} \sum_{w=1}^{W} \sum_{d=1}^{D} T D_{w d}\right)+\sum_{w}^{W} h_{w} C_{w} D l+\sum_{m=1}^{M} e_{m} C_{m}^{s p}+\sum_{m=1}^{M} s h_{m} C_{m}^{s h}
$$

subject to:

$$
\begin{aligned}
& \sum_{w=1}^{W} z_{m w d} \leq 1 \quad \forall m=1, \ldots, M, \quad \forall d=1, \ldots, D \\
& u_{m d}=\sum_{w=1}^{W} z_{m w d}, \quad \forall m=1, \ldots, M, \forall d=1, \ldots, D \\
& z_{m w d} \leq h_{w}, \quad \forall m=1, \ldots, M, \quad \forall w=1, \ldots, W, \forall d=1, \ldots, D \\
& \left.\begin{array}{l}
\sum_{i=1}^{M} x_{w d i m} \leq h_{w} \\
\sum_{j=1}^{M} x_{w d m j} \leq h_{w}
\end{array}\right\} \quad \forall m=1, \ldots, M, \forall w=1, \ldots, W, \forall d=1, \ldots, D \\
& x_{w d i j} \leq h_{w} \quad \forall m=1, \ldots, M, \forall w=1, \ldots, W, \forall d=1, \ldots, D \\
& T D_{w d}=\sum_{i=1}^{n} \sum_{j=1}^{n} d_{i s t} x_{i j} x_{w i j}, \quad \forall w=1, \ldots, W, \forall d=1, \ldots, D \\
& \sum_{m}^{M} d_{m} z_{m w d}+\frac{1}{S} \sum_{i=1}^{n} \sum_{j=1}^{n} d_{i s t} x_{i j} x_{w d i j} \leq l_{d} \quad \forall w \quad \forall d=1, \ldots, D \\
& \sum_{j=1}^{M} x_{w d i j}=z_{i w d}, \quad i=1, \ldots, M \\
& \left.\sum_{i=1}^{M} x_{w d i j}=z_{j w d}, \quad j=1, \ldots, M\right) \\
& \left.\begin{array}{c}
Q_{w d i}-Q_{w d j}+M x_{w d i j} \leq(M-1) \\
i=2, \ldots, M, j=2, \ldots, M: i \neq j
\end{array}\right) \\
& \forall w=1, \ldots, W, d=1, \ldots, D, \\
& e_{m} \geq \sum_{d=1}^{D} u_{m d}-f_{m} \\
& e_{m} \leq \widehat{M} y_{m}^{1} \\
& \left.e_{m} \leq \sum_{d=1}^{D} u_{m d}-f_{m}+\widehat{M}\left(1-y_{m}^{1}\right)\right\} \\
& \forall m=1, \ldots, M \\
& s h_{m} \geq 1-\left(\sum_{d=1}^{D} u_{m d}\right) / f_{m} \\
& s h_{m} \leq \widehat{M} y_{m}^{2} \\
& \left.s h_{m} \leq 1-\left(\sum_{d=1}^{D} u_{m d}\right) / f_{m}+\widehat{M} \times\left(1-y_{m}^{2}\right)\right\} \\
& \forall m=1, \ldots, M
\end{aligned}
$$




$$
\begin{aligned}
& \left.z_{1 w d}=1\right\} \\
& \left.z_{2 w d}=1\right\} \\
& \forall w=1, \ldots, W, \forall d=1, \ldots, D \\
& \left.u_{1 d}=1\right\} \\
& \left.u_{2 d}=1\right\} \\
& \forall d=1, \ldots, D
\end{aligned}
$$

where the input parameters are:

- $d=1, \ldots, D$ days in the planning period.

- $m=1, \ldots, M$ tasks.

- $w=1, \ldots, W$ teams.

- $\quad d_{m}$ : duration of task $m$.

- dist $_{i j}$ the distance between task sites of $i$ and $j$.

- $\quad f_{m}$ : nominal frequency of task $m$.

- $\quad C_{m}^{s p}$ : cost of spare parts of task $m$.

- $\quad C_{m}^{s h}$ : cost of shortage in service level of task $m$.

- $S$ : travel speed of the maintenance teams.

- $R$ : cost rate of traveled unit distance

- $C_{w}$ : team cost per hr.

- $l_{d}$ : duration of day $d$.

- $\widehat{M}$ : significantly large number, where $M \gg$ nand $\gg \max (f)$.

\section{Decision variables:}

- $\quad z_{m w d}=\left\{\begin{array}{l}1, \text { if maintenance task } m \text { is performed by pda team } w \text { on dayd } \\ 0,\end{array}\right.$

- $Q_{w d i}$ : auxiliary variable to eliminate sub tours.

- $e_{m}$ : extra number of tasks.

- $s h_{m}$ : Shortage percent of tasks, i.e., 1-service level.

- $\quad u_{m d}=\left\{\begin{array}{lr}1, & \text { if maintenance task } m \text { is performed on dayd } \\ 0, & \text { otherwise }\end{array}\right.$

- $\quad x_{w d i j}= \begin{cases}1, & \text { if team } w \text { moves from a site } i \text { to site } j \text { in dayd } \\ 0, & \text { otherwise }\end{cases}$

- $h_{w}=\{1, \quad$ if team $w$ is hired

- $y_{1}=\left\{\begin{array}{l}1, \\ 0, \quad \text { if the number of extra tasks }>0\end{array}\right.$

- $y_{1}= \begin{cases}1, & \text { otherwise }\end{cases}$

- $y_{2}=\left\{\begin{array}{lr}1, & \text { if the percentage of short tasks }>0 \\ 0, & \text { otherwise }\end{array}\right.$

The objective function in Eq. (5) consists of four segments, the first denotes the cost of traveling between the different sites at a speed of $S$ and a cost rate of $R$, where the traveled distance is given by constraint (11) which sums the traveled distance between the task sites over the selected routes of all teams. Note that each team will have a specific route that varies day to day, hence, $W \times D$ optimal routes will result in this model. The second expression in the objective function is the cost of hired teams $/ \mathrm{hr}$ times the number of working hours in the planning period. The third term is the cost of performing extra tasks of $m$, which only entails the cost of additional spare parts here. Note that spare parts of the tasks that fall within the nominal frequency are paid by the client, while those above this limit are paid by the service provider. Almost similar to the notion of extra tasks, shortage in tasks is penalized by the percentage, where $s h_{m}$ is the percentage of the short number of $m$. The percentage is simply found by Eq. (3). In many service contracts, especially in commercial buildings, penalties are typically applied upon low service levels rather than the number of unmet tasks. Usually both clients and service providers prefer service level penalties as compared to other fine policies.

However, in formulating $e_{m}$ and $s h_{m}$ one should be careful of negative values. Therefore, the purpose of the constraint set in Eq. (14) and Eq. (15) which use the BigM trick is to avoid negative numbers that will mislead the solver. With these constraints, we maintain the status of either $e_{m}$ or $s h_{m}$ can have a positive value, but not both. 
Constraint (6) states that a task can be done at most once a day. Due to tractable modeling, we can easily verify that $z_{m w d}=$ $\sum_{i=1}^{M} x_{w d i m}$ and $z_{m w d}=\sum_{j=1}^{M} x_{w d m j}$, hence, constraint (6) can be modeled in different ways such as $\sum_{w=1}^{W} \sum_{j=1}^{M} x_{w d m j} \leq 1$. To get the daily schedule of each team, summing the matrix $z$ over $w$ will yield such an output that is given by $u_{m d}$ in constraint (7). Consequently, a $u_{m d}$ value of 1 means that task $m$ has to be performed in day $d$, and " 0 " otherwise.

Since a hired team is assigned a value of $h_{w}=1$ and " 0 " otherwise, the constraint of (8) puts more restriction on the value of $z$, where if a team is not hired, intuitively $z$ must have a value of zero. The same applies to the constraints in (9) and also to the constraint in Eq. (10) since $z$ is equivalent to both of $\sum_{j=1}^{M} x_{w d i j}$ and $\sum_{i=1}^{M} x_{w d i m}$. The constraint in (10) shows that no sites associated with a team $w$ will be visited if the team is not hired (i.e., $h_{w}=0$ ). For the time constraint in (12), the duration of the performed tasks by each team in addition to the travelling time should not exceed the daytime given by $l_{d}$. While day times can have different lengths, however, in our experiments we use 8hrs per day. The constraints set of Eq. (13) identifies the routes of those visited sites, where the first two constraints apply only when $z=1$ (i.e., visited sites only). The first constraint states that only one outward link should depart from site $i$. Similarly, the second constraint shows that only one inward link should go into task $j$. The last constraint is for eliminating sub-tours (multiple separate cycles) where $Q_{w d i}$ is an integer number (please refer to TSP for more details). In contrast to the traditional TSP in which all the sites have to be visited, the two equalities mentioned above only apply to the set of tasks planned to be performed, however, when $z_{i w d}=0$, the constraints and the routes coming in and out of task $i$ are no longer valid, i.e., $x_{w d i} \bullet=0$, similarly, if $z_{j w d}=0$, then $x_{w d \bullet j}=0$. To avoid infeasibility in the resulting solution due to teams that are not hired, one may want to consider adding the condition $x_{w d 12}=$ $1, \forall w, \forall d$ when coding the model to force visiting the maintenance depot by all teams. The constraint sets of Eq. (14) and Eq. (15) avoid negative values of both extra and short tasks as illustrated above. Of note, the classical formulation of the TSP does not work for networks of less than two sites which justifies the beginning by "2" in some constraint indices. Moreover, since the start/end location must be visited every day, this implies the addition of more constraints to force visiting the start/end location by all teams on a daily basis as shown in the constraints of (16) and (17). By minimizing the cost function, the model should aid in the following:

1. Finding the necessary number of teams that is capable of carrying out the tasks.

2. Establishing the schedule of tasks that conforms to the settings and given frequencies.

3. Assigning teams to tasks.

4. Identifying the optimal daily routes of every team.

It is worth noting that the computational complexity resulting from finding the minimum routes of each team, each day is the most challenging issue in this model due to the dramatic increase of variables. While it is agreed that the TSP problem is NPhard, to demonstrate the level of computational challenge of the problem here, the number of times a TSP-variant is conducted in each solution trial will be $M \times W \times D$ for a set of $M$ tasks, initial $W$ teams, and $D$ days. This shows the computational difficulty this problem presents while seeking the optimal schedule and routes. Some interesting features that classify the distinctiveness of our model are as follows; the model reduces to a typical travel salesman problem when only one-day planning is considered with a restriction for the total stay at each repair site, on the other hand, the model reduces time-constrained selective maintenance if the task sites are not spatially distributed (i.e., zero distances). Such different scenarios are demonstrated by the experiments in section 5 .

\section{Experimental Results}

Coded using AMPL (A Mathematical Programming Language) with CPLEX as our solver of choice, the model presented in (5)-(17) which is characterized by linear objective function and constraints was validated using different instances taken from real service providers in UAE. The code was tested on Intel i7 Core, $2.11 \mathrm{GHz}$ processor, and 16 GB RAM. First, for better visualization of the model solutions, we begin with a purposely simplistic example that can be extended to more advanced instances. Here, we consider 5 tasks which should be performed over two days with a required frequency of two each. This results in 7 indexed sites including the start/end point. Table 1 presents the tasks and team costs while Fig. 2- $a$ demonstrates the physical sites of the tasks where the origin is the start/end point of the maintenance teams. Euclidean distances are considered in this example.

Table 1

Task and team costs. Note both that 1 and 2 refer to the site of maintenance shop.

\begin{tabular}{ccccccccccc}
\hline Task & 1 & 2 & 3 & 4 & 5 & 6 & 7 & & Team $w$ & $C_{w}$ \\
\hline$C_{m}^{s p}$ & - & - & 10 & 5 & 30 & 20 & 11 & & 1 \\
$d_{m}$ & - & - & 2.5 & 1 & 2 & 0.5 & 1.5 & 20 & 30 \\
$C_{m}^{s h}$ & - & - & 8270 & 3680 & 1680 & 8250 & 3540 & 3 & 25 \\
\hline
\end{tabular}

First, we test the model by infinite travel speed, which is, in effect, the same assumption of zero distances between the sites. The results in Table 2 show that only one team is required to performed the tasks over the two days at a cost of 320AED, which is simply the cost of hiring the team, this is: 8 hrs $\times 2$ days $\times 20 \mathrm{AED} / \mathrm{hr}$ (1AED is equivalent to $\$ 0.272$ at the time of the study). Note that with zero travel time, the selection of the path for which the tasks will be performed is irrelevant as all paths 
have zero times. However, as the travel speed begins to decrease, the impact of travel time begins to appear. Note that, a speed of $50 \mathrm{~km} / \mathrm{hr}$ is still fast enough to perform the tasks by one team that follows the shortest route which is $42.3 \mathrm{~km}$ long. In fact, this distance represents the typical solution of the TSP for the seven sites shown in Fig. 2. While the utilization and efficiency in Table 2 are the same at an infinite speed (owed to inexistence of travel time), it will not be the case for other speeds, as at lower speeds, the travel time begins to consume from the teams' available time. Hence, at a speed of 50, although the travel distance is the same as the first row, yet, the utilization is different because of the additional 0.846hrs consumed in traveling. Although no short and extra jobs are performed at a speed of 50, the optimal cost (cost of teams + traveling at a rate of $50 \mathrm{AED} / \mathrm{hr}$ ) is $20 \times 16+42.3 \times 50 / 50=362.3 \mathrm{AED}$. Similarly, as it takes two to tango, it also takes two teams to perform the tasks when the speed drops to $5 \mathrm{~km} / \mathrm{hr}$. Here, $6.616+6.616=13.232 \mathrm{hrs}$ are consumed in traveling over the two days, which lowers the utilization to significant numbers. Conversely, if the travel time of each team is considered as a working time, the efficiency will be around $88.22 \%$ for both teams. Three teams are required if the travel speed drops to $3 \mathrm{~km} / \mathrm{hr}$ which can be observed inside shopping malls. While the traveled distance decreased by having more teams, the travel time tends to increase due to slower speeds. Clearly, at such a low speed, the time utilization will be significantly low.

Table 2

Results of first instance (5 tasks, 1-3 teams, and 2 days).

\begin{tabular}{|c|c|c|c|c|c|c|c|c|c|c|c|c|}
\hline $\begin{array}{l}\text { Travel } \\
\text { speed } \\
(\mathrm{km} / \mathrm{hr})\end{array}$ & 苨 & Team & 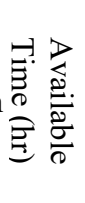 & 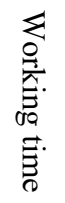 & 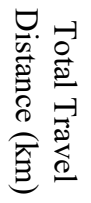 & 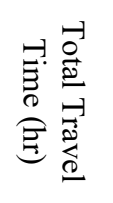 & $\begin{array}{c}\text { Time } \\
\text { Utilization }\end{array}$ & $\begin{array}{c}\text { Team } \\
\text { Efficiency }\end{array}$ & Resulting Route & $\begin{array}{l}\text { (no.) } \\
\text { Short } \\
\text { tasks }\end{array}$ & $\begin{array}{l}\text { Extra } \\
\text { tasks }\end{array}$ & Cost \\
\hline$\infty \dagger$ & 1 & $\mathrm{~A}$ & 16 & 15 & 42.30 & 0 & 0.93800 & 0.93800 & $\dagger$ & 0 & 0 & 320 \\
\hline 50 & 1 & A & 16 & 15 & 42.30 & 0.846 & 0.9375 & 0.99037 & $\begin{array}{l}\text { Day 1: } 1-3-4-5-6-7-2 \\
\text { Day 2: } 1-7-6-5-4-3-2\end{array}$ & 0 & 0 & 362.3 \\
\hline \multirow{2}{*}{5} & \multirow{2}{*}{2} & A & 16 & 7.5 & 33.08 & 6.616 & 0.46875 & 0.88225 & $\begin{array}{l}\text { Day 1: } 1-7-3-2 \\
\text { Day 2: } 1-6-5-4-2\end{array}$ & \multirow{2}{*}{0} & \multirow{2}{*}{0} & \multirow{2}{*}{1381.6} \\
\hline & & $\mathrm{B}$ & 16 & 7.5 & 33.08 & 6.616 & 0.46875 & 0.88225 & $\begin{array}{l}\text { Day 1: } 1-6-5-4-2 \\
\text { Day } 2: 1-7-3-2\end{array}$ & & & \\
\hline \multirow{3}{*}{3} & \multirow{3}{*}{3} & A & 16 & 4 & 26.62 & 8.8733 & 0.25 & 0.80458 & $\begin{array}{l}\text { Day 1: } 1-6-7-2 \\
\text { Day } 2: 1-5-2\end{array}$ & \multirow{3}{*}{3,4} & \multirow{3}{*}{0} & \multirow{3}{*}{8517} \\
\hline & & B & 16 & 3.5 & 32.7 & 10.9 & 0.1875 & 0.86875 & $\begin{array}{l}\text { Day 1: } 1-5-2 \\
\text { Day 2: } 1-4-2\end{array}$ & & & \\
\hline & & $\mathrm{C}$ & 16 & 4 & 21.2 & 7.0667 & 0.28125 & 0.72292 & $\begin{array}{l}\text { Day } 1: 1-3-2 \\
\text { Day } 2: 1-6-7-2\end{array}$ & & & \\
\hline
\end{tabular}

$\uparrow:$ due to infinite speed, the travel time is " 0 ", hence, the sequence of tasks does not matter.

The cost objective function can be easily computed upon finding the routes and the number of extra/short tasks. For instance, consider the last case in Table 1 , the cost of 3 teams is $20 \times 16 \mathrm{hrs}+25 \times 16+30 \times 16=1200$ AED. Traveling at the given speed will cost $\frac{(26.62+32.7+21.2) \mathrm{km}}{3 \mathrm{~km} / \mathrm{hr}} \times 50 \mathrm{AED} / \mathrm{hr}=1342 \mathrm{AED}$, the cost of the two short jobs $\left(3\right.$ and 4 here) is $\frac{1}{2} \times 8270+$ $\frac{1}{2} \times 3680=5975$ AED and the total of these costs will sum to 8517.002 . Fig. 2 shows the resulting paths of day 1 of the three cases shown in Table 1.

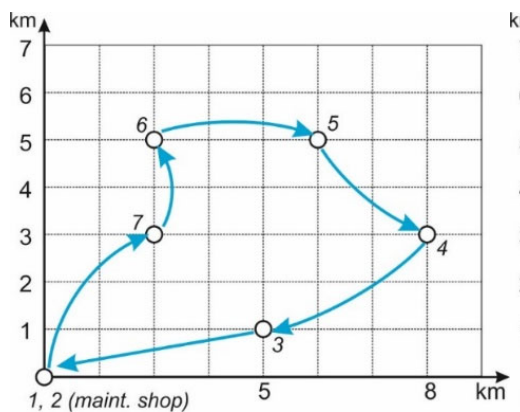

(a)

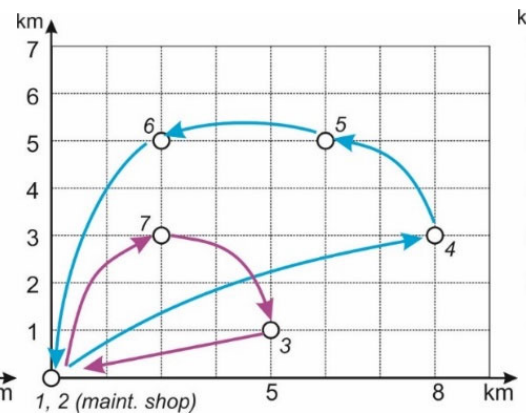

(b)

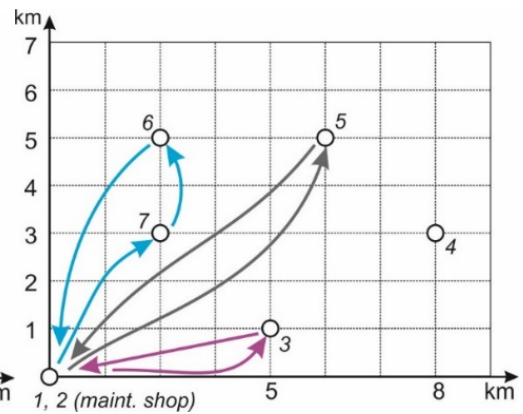

(c)

Fig. 2. a) The routes of the optimal number of teams on the first day at a speed of $50 \mathrm{~km} / \mathrm{hr}$. $b$ ) The routes of the optimal number of teams on the first day at a speed of $5 \mathrm{~km} / \mathrm{hr} . c$ ) The routes of the optimal number of teams on the first day at a speed of $2.5 \mathrm{~km} / \mathrm{hr}$ 
Our second scenario considers a realistic yet simple example to demonstrate the impact of travel time on maintenance tasks of geographical distribution. Here, a maximum of three teams are considered to perform a set of 10 tasks over a period of one week. The data of this example are shown in Table 3. For instance, the cost of task 3 will be 10,000 AED if not done at all in this planning period. Therefore, if $50 \%$ of the required frequency of this task is accomplished, the cost will be 5,000AED. The shortage cost will be zero once the required frequency is accomplished. On the other hand, any extra job will incur an additional spare part cost in addition to working team cost, if for example task 10 is performed 8 times instead of 7 , an additional cost of 20AED is incurred. Each team costs 25 AED per hr. Note that, in our model, we do not always assume symmetric distances, meaning that the round-trip distances are not necessarily the same.

Table 3

An instance of 10 tasks, 3 teams, and one-week period. The costs are in AED.

\begin{tabular}{|c|c|c|c|c|c|c|c|c|c|c|c|c|c|c|}
\hline \multirow[b]{2}{*}{ Task } & \multirow[b]{2}{*}{$d_{i}$} & \multirow[b]{2}{*}{$f_{m}$} & \multirow[b]{2}{*}{$C_{m}^{s p}$} & \multirow[b]{2}{*}{$C_{m}^{s h}$} & \multicolumn{9}{|c|}{$d_{i s t} t_{i j}$} & \multirow[b]{2}{*}{10} \\
\hline & & & & & 1 & 2 & 3 & 4 & 5 & 6 & 7 & 8 & 9 & \\
\hline 1 & 0 & 7 & 0 & 0 & 0 & 0 & 6.1 & 2 & 2.8 & 4.4 & 2.5 & 8.4 & 4.5 & 3.5 \\
\hline 2 & 0 & 7 & 0 & 0 & 0 & 0 & 6.1 & 2 & 2.8 & 4.4 & 2.5 & 8.4 & 4.5 & 3.5 \\
\hline 3 & 1.5 & 7 & 100 & 10,000 & 1.9 & 1.9 & 9.9 & 3 & 7.9 & 2.6 & 3.6 & 7.4 & 6.1 & 5.4 \\
\hline 4 & 1.25 & 7 & 50 & 12,000 & 2.2 & 2.2 & 9.8 & 9.2 & 6.2 & 4.8 & 6.2 & 9.3 & 7.3 & 5.1 \\
\hline 5 & 1 & 7 & 55 & 15,000 & 0.5 & 0.5 & 3.3 & 1.8 & 7.1 & 7.9 & 4.9 & 3.9 & 2.4 & 6.9 \\
\hline 6 & 3.5 & 7 & 60 & 8,000 & 8.8 & 8.8 & 6 & 4.4 & 2.5 & 7.2 & 8.3 & 5.9 & 7.8 & 0.3 \\
\hline 7 & 0.5 & 7 & 60 & 12,000 & 9.8 & 9.8 & 9.2 & 5.2 & 0.5 & 5.3 & 7.4 & 1.9 & 4.2 & 6.1 \\
\hline 8 & 0.5 & 7 & 150 & 15,000 & 3.9 & 3.9 & 8.6 & 1 & 1.1 & 8.6 & 8.6 & 3.1 & 6.9 & 0.4 \\
\hline 9 & 2 & 7 & 220 & 6,000 & 5.8 & 5.8 & 7.4 & 5 & 5.9 & 1.9 & 6 & 2.7 & 1.8 & 8.9 \\
\hline 10 & 3.0 & 7 & 20 & 14,000 & 4.2 & 4.2 & 4.9 & 8.6 & 5.8 & 5.5 & 2.4 & 2.8 & 2.2 & 6.1 \\
\hline Teams & $C_{w}$ & & & & $R=$ & 1 & $\mathrm{AED} / \mathrm{hr}$ & & & & & & & \\
\hline 1 & 25 & & & & & & & & & & & & & \\
\hline 2 & 25 & & & & & & & & & & & & & \\
\hline 3 & 25 & & & & & & & & & & & & & \\
\hline
\end{tabular}

The total time required to perform the tasks is $\sum_{i=1}^{10} f_{i} d_{i}=92.75 \mathrm{hrs}$. While this is the needed time to perform the job with no constraints, in reality, the tasks will consume more than this due to traveling between tasks, contradiction of team schedules and the limit of 8 working hours per day. Using the proposed model, it will be possible to find the number of workers and team schedules that satisfy the given constraints yet demonstrate the lowest cost. For benchmarking, first, we consider zero distances between task sites. Under this assumption, the resulting cost of the above scenario is 2800AED with no short or excess jobs experienced. The total number of hired teams is 2 with efficiencies of $74.5 \%$ and $91 \%$, respectively. Clearly, this maintenance schedule cannot be performed by one team and no other schedule can demonstrate lower cost. To reflect reality, the start and end sites must be visited every day, which are in essence the assembly points of the workers. Fig. 3 shows a Gant-like schedule of the tasks for the zero distance matrix.

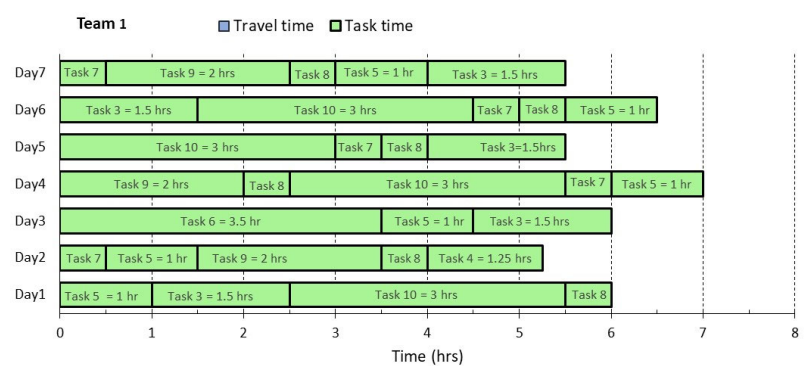

Fig. 3-a. The daily schedule of team 1 at zero distances (efficiency $=74.5 \%$ )

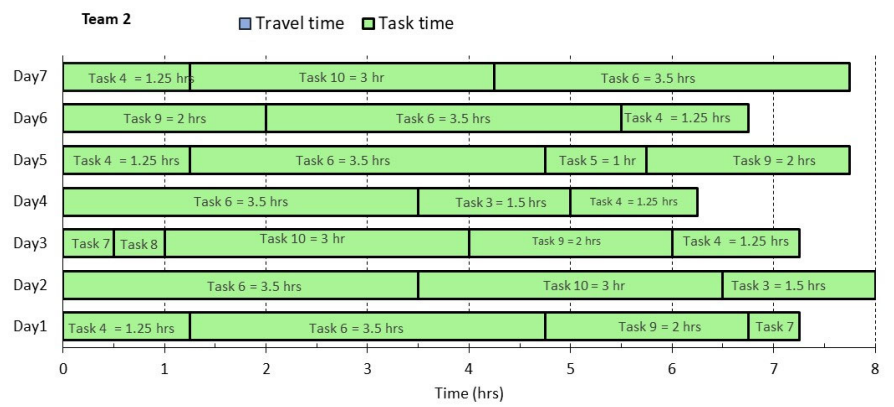

Fig. 3-b. The schedule of team 2 at zero distances, (efficiency=91\%) 
At a slow travel speed, say $15 \mathrm{~km} / \mathrm{hr}$, two teams are still enough to perform the tasks without shortage. At this speed, the minimum cost is 2813.887 AED. The average utilization is $82.8 \%$ due to travel time, while the team efficiencies are $94.47 \%$ and $95.95 \%$, respectively. Fig. 4 shows the team schedule over seven days. The emerging gaps represent the time consumed in travelling. For example, on day 6 , team 1 has to travel first to task 9 then $8,10,3$, respectively, and lastly back to the maintenance shop. The time consumed in traveling of this team for the same day and speed is $0.961 \mathrm{hrs}$ detailed as follows: start-to-9:0.3hrs, 9-to-8:0.180hrs, 8-to-10:0.027hrs, 10-to-3:0.327hrs and lastly, 3-to-home: $0.127 \mathrm{hrs}$.

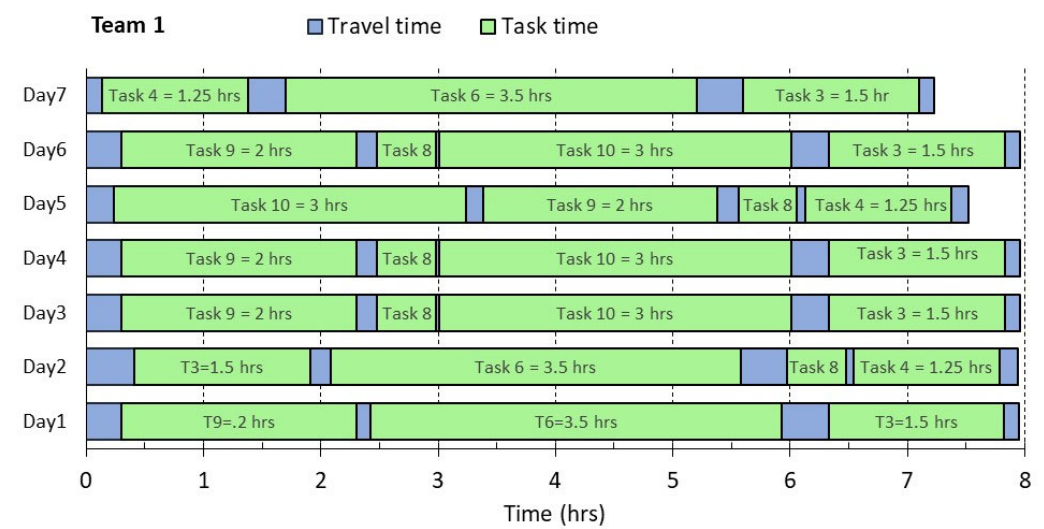

Fig. 4-a. The schedule of team 1 at a speed of $15 \mathrm{~km} / \mathrm{hr}$ (efficiency $=94.47 \%$ )

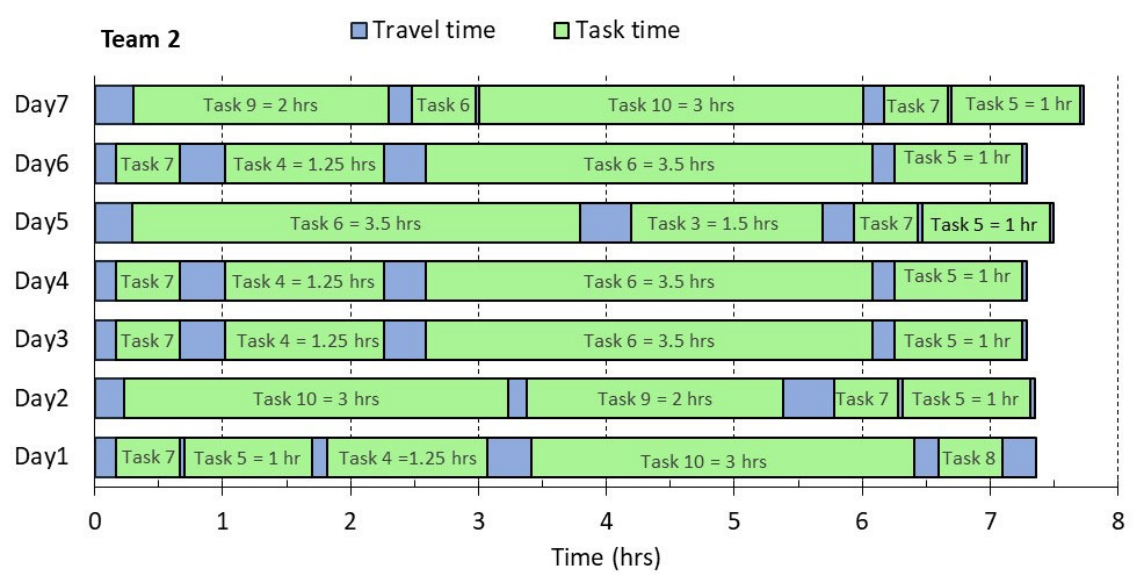

Fig. 4-b. The schedule of team 2 at a speed of $15 \mathrm{~km} / \mathrm{hr}$ (efficiency $=95.95 \%)$

At a walking or electrical shuttle speed, the travel time will consume more of the available 8 hrs per team. Therefore, at a speed of $5 \mathrm{~km} / \mathrm{hr}$, no less than 3 teams can perform the tasks. In this case, upon finding the optimal schedule, the total traveled distance of the 3 teams is $228.5 \mathrm{~km}$ which takes $45.7 \mathrm{hrs}$ of travel time. The resulting cost of this scenario is $4246.225 \mathrm{AED}$. The efficiencies of the three teams are $74.9 \%, 81.78 \%, 90.52 \%$, respectively. The schedules are shown in Fig. 5. Notably, the travel time is consuming a significant proportion of the available team times leading to low utilization.

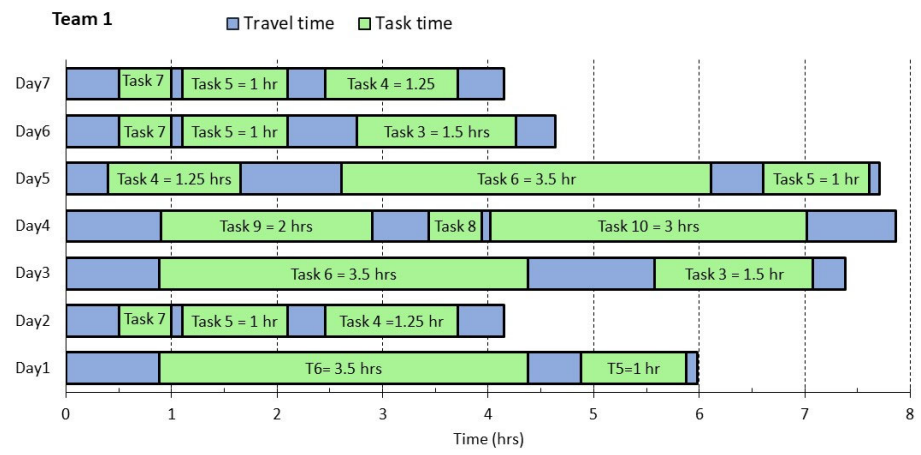

Fig. 5-a. The schedule of team 1 at a speed of $5 \mathrm{~km} / \mathrm{hr}($ efficiency $=74.9 \%)$ 


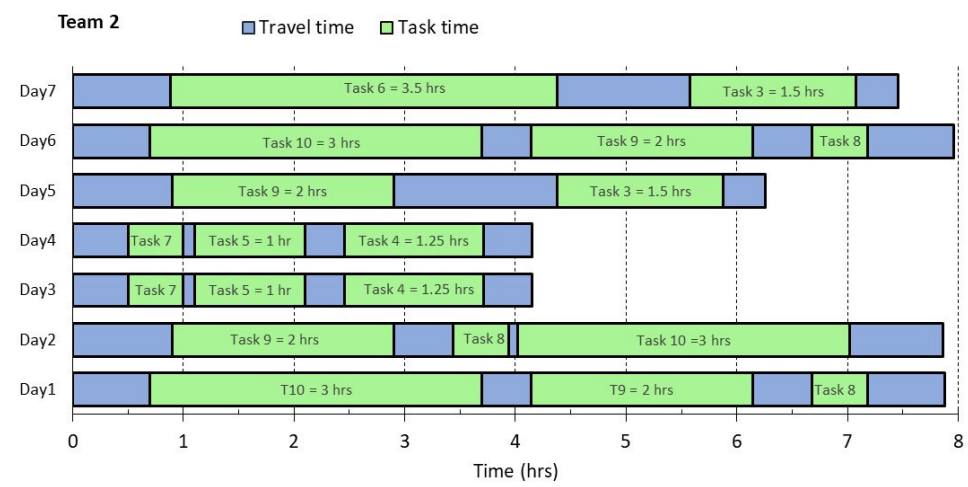

Fig. 5-b. The schedule of team 2 at a speed of $5 \mathrm{~km} / \mathrm{hr}($ efficiency $=81.78 \%)$.

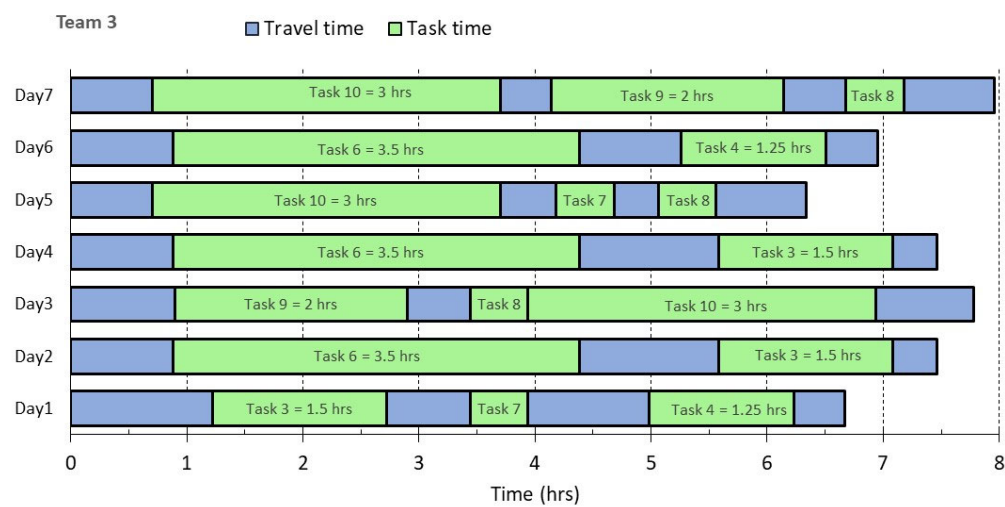

Fig. 5-c. The schedule of team 3 at a speed of $5 \mathrm{~km} / \mathrm{hr}($ efficiency $=90.52 \%)$.

In the next third scenario, the previous example is extended to a maximum of 5 teams and 30 days in which 10 tasks of new durations should be scheduled according to the required frequencies shown in Table 4. Table 5 demonstrates the resulting teams and the associated cost and team efficiency. Note that the time required to finish the task in a back-to-back manner with no constraints is $503 \mathrm{hrs}$ which can be found by $\sum_{i}^{M} f_{i} \times d_{i}$. Accordingly, since a team can put as many as $8 \times 30=240 \mathrm{hrs}$ in the whole period, there is no possible schedule that can be put in place to finish the tasks by only one team.

Table 4

Data for the third scenario

\begin{tabular}{|c|c|c|c|c|c|c|c|c|c|c|c|c|c|c|}
\hline \multirow[b]{2}{*}{ Task no } & \multirow[b]{2}{*}{$d_{m}(\mathrm{hr})$} & \multirow[b]{2}{*}{$f_{m}$} & \multirow[b]{2}{*}{$C_{m}^{s p}$} & \multirow[b]{2}{*}{$C_{m}^{s h}$} & \multicolumn{10}{|c|}{$\operatorname{dist}_{i j}$} \\
\hline & & & & & 1 & 2 & 3 & 4 & 5 & 6 & 7 & 8 & 9 & 10 \\
\hline 1 & 0 & 30 & - & 0 & 0 & 0 & 0.8 & 3.4 & 2.2 & 6.2 & 1.1 & 0.3 & 8.5 & 5.7 \\
\hline 2 & 0 & 30 & - & 0 & 0 & 0 & 0.8 & 3.4 & 2.2 & 6.2 & 1.1 & 0.3 & 8.5 & 5.7 \\
\hline 3 & 3.5 & 35 & 100 & 82.7 & 2.5 & 2.5 & 0 & 8.8 & 6.8 & 6.4 & 7.7 & 5.8 & 6.2 & 7.2 \\
\hline 4 & 3 & 10 & 50 & 36.8 & 9.9 & 9.2 & 2.7 & 0 & 5.7 & 1.5 & 9.7 & 6.7 & 8.4 & 0.19 \\
\hline 5 & 2 & 25 & 300 & 168 & 7.2 & 7.2 & 5.9 & 4.3 & 0 & 7.8 & 3.2 & 4.2 & 7.7 & 7.1 \\
\hline 6 & 1 & 15 & 200 & 82.5 & 3.7 & 3.7 & 6.7 & 0.8 & 4.8 & 0 & 8.8 & 6.2 & 1.1 & 6.8 \\
\hline 7 & 2.5 & 30 & 110 & 35.4 & 1.8 & 1.8 & 1.5 & 3.8 & 1.2 & 5.4 & 0 & 7.2 & 6.3 & 9.1 \\
\hline 8 & 4 & 70 & 150 & 139.1 & 6.2 & 6.2 & 3.8 & 3.4 & 4.6 & 9.7 & 9.9 & 0 & 1.8 & 8.4 \\
\hline 9 & 3.5 & 40 & 220 & 153.8 & 1.9 & 1.9 & 7.7 & 0.7 & 6.6 & 5.7 & 0.5 & 1.3 & 0 & 2.9 \\
\hline 10 & 0.5 & 6 & 130 & 73.6 & 7.7 & 7.7 & 6.1 & 5.9 & 2.6 & 4.2 & 6.5 & 8.3 & 2.6 & 0 \\
\hline Team & 1 & 2 & 3 & 4 & 5 & & & & & & & & & \\
\hline$C_{w}$ & 20 & 30 & 25 & 21 & 22 & & & & & & & & & \\
\hline
\end{tabular}

The results in Table 5 show that a minimum of 3 teams can present a schedule that is sufficient to perform the tasks. At a speed of $10 \mathrm{~km} / \mathrm{hr}, 4$ teams are required while 5 teams are needed at $2.5 \mathrm{~km} / \mathrm{hr}$. The time utilization in the third column is getting lower owed to the increase in travel time, however, the team efficiencies are higher since the travel time is considered as a productive time in the efficiency calculations. Nevertheless, the remaining unutilized time of each team results because 
of other physical constraints such as the daily remaining time of each team which does not fit any remaining tasks that still need to be done. This in addition to the discrete nature (integer number) of hired teams, as by assumption, teams cannot be partially employed.

Table 5

An instance of 10 tasks, 5 teams, and 30 days period. The costs are in AED.

\begin{tabular}{ccccccccc}
\hline $\begin{array}{c}\text { Travel } \\
\text { speed } \\
(\mathrm{km} / \mathrm{hr})\end{array}$ & $\begin{array}{c}\text { Optimal } \\
\text { number of } \\
\text { teams }\end{array}$ & $\begin{array}{c}\text { Time } \\
\text { Utilization }\end{array}$ & $\begin{array}{c}\text { Average } \\
\text { Team } \\
\text { Efficiency }\end{array}$ & $\begin{array}{c}\text { Cost } \\
\text { AED }\end{array}$ & $\begin{array}{c}\text { Total Travel } \\
\text { Distance }(\mathrm{km})\end{array}$ & $\begin{array}{c}\text { Total } \\
\text { Travel } \\
\text { time }(\mathrm{hr})\end{array}$ & $\begin{array}{c}\text { Short } \\
\text { tasks }\end{array}$ & $\begin{array}{c}\text { Extra } \\
\text { tasks }\end{array}$ \\
\hline$\infty^{\dagger}$ & 3 & 0.699 & 0.699 & 15120.00 & 900.700 & 0 & 0 & 0 \\
10 & 4 & 0.524 & 0.592 & 24389.78 & 653.950 & 65.395 & 0 & 0 \\
2.5 & 5 & 0.419 & 0.742 & 47678.04 & 967.900 & 503.000 & 0 & 0 \\
\hline
\end{tabular}

$\dagger$ : Equivalent to zero distances. The rate $R=50 \mathrm{AED} / \mathrm{hr}$.

Clearly, the cost will increase by lower speeds due to more hiring and possibly more shortage experienced. The behavior of the travelled distance is a little unpredictable as we decrease the speed since the fewer the number of sites visited; the less distance traveled by each team. However, regardless of the behavior of the resulting traveled distance, the travel time will significantly increase by lower speeds. With low speeds, shortages in some tasks begin to arise owed to daily working hours.

Next, we demonstrate a larger instance where 10 maintenance tasks are to be scheduled over a period of 60 days. The instance data is shown in Table 6 . We initially choose to begin with 8 teams, then the model will find the optimal number of teams and schedules. Upon solving this instance, it was found only 3 teams are sufficient to do the work as illustrated in Table 7. The total traveled distances by each of the three teams over the 60 days are $471.2 \mathrm{~km}, 509.8 \mathrm{~km}$ and $538.7 \mathrm{~km}$, respectively, with a total of $1,519.7 \mathrm{~km}$ consuming a travel time of $1,519.7 / 5=303.94 \mathrm{hrs}$ at a speed of $5 \mathrm{~km} / \mathrm{hr}$. The resulting team efficiencies are $81.77 \%, 87.18 \%$, and $86.50 \%$, respectively, for the three teams. The slack time that has not been utilized averages to $1.19 \mathrm{hrs}$ per day per team due to the non-preemptive assumption which entails not proceeding in any additional task if the task cannot be completed within the remaining time of the day and also the integer number of teams. The number of short tasks is 8 (6 times for task 4 and 2 times for task 6) and 0 extra jobs. Table 5 shows a sample of the results.

Table 6

Data for the fourth scenario. All costs in AED.

\begin{tabular}{|c|c|c|c|c|c|c|c|c|c|c|c|c|c|c|}
\hline \multirow[b]{2}{*}{ Task no } & \multirow[b]{2}{*}{$d_{m}(\mathrm{hr})$} & \multirow[b]{2}{*}{$f_{m}$} & \multirow[b]{2}{*}{$C_{m}^{s p}$} & \multirow[b]{2}{*}{$C_{m}^{s h}$} & \multicolumn{10}{|c|}{$d_{i s t} t_{i j}$} \\
\hline & & & & & 1 & 2 & 3 & 4 & 5 & 6 & 7 & 8 & 9 & 10 \\
\hline 1 & 0 & 60 & - & - & 0 & 0 & 0.4 & 0.9 & 5.4 & 2.4 & 3.8 & 6.7 & 8.5 & 8.9 \\
\hline 2 & 0 & 60 & - & - & 0 & 0 & 0.4 & 0.9 & 5.4 & 2.4 & 3.8 & 6.7 & 8.5 & 8.9 \\
\hline 3 & 1 & 45 & 10 & 300 & 7.6 & 7.6 & 0 & 6.2 & 5.3 & 5.5 & 8 & 1.9 & 0.5 & 6 \\
\hline 4 & 2.5 & 60 & 5 & 700 & 4.2 & 4.2 & 2.8 & 0 & 5.1 & 7.5 & 2.5 & 5.5 & 9.5 & 0.2 \\
\hline 5 & 1 & 35 & 30 & 900 & 9.1 & 9.1 & 0.7 & 10 & 0 & 1.1 & 6.1 & 9.5 & 9 & 6 \\
\hline 6 & 4.5 & 60 & 20 & 1000 & 1.6 & 1.6 & 9.4 & 4.5 & 6.6 & 0 & 5 & 4.3 & 9 & 6 \\
\hline 7 & 3 & 55 & 11 & 1000 & 6.6 & 6.6 & 2.6 & 1.3 & 5.1 & 8.7 & 0 & 3.1 & 8 & 4.9 \\
\hline 8 & 3.25 & 15 & 15 & 400 & 8.5 & 8.5 & 8.2 & 5.8 & 9.4 & 9.3 & 7 & 0 & 6.5 & 5.6 \\
\hline 9 & 2.5 & 43 & 22 & 600 & 5.7 & 5.7 & 6.4 & 3.1 & 2.3 & 7.4 & 7.6 & 4.1 & 0 & 0.8 \\
\hline 10 & 2.5 & 50 & 13 & 700 & 3.8 & 3.8 & 9.6 & 0.9 & 5.1 & 9.6 & 5 & 2.9 & 0.8 & 0 \\
\hline Team & 1 & 2 & 3 & & & & & & & & & & & \\
\hline$C_{w}$ & 16 & 15 & 14 & & & & & & & & & & & \\
\hline
\end{tabular}

Table 7

Results of 10 tasks and 60 days maintenance schedule

\begin{tabular}{ll}
\hline Parameters/Outputs & \\
\hline Rate & $10 \mathrm{AED} / \mathrm{hr}$ \\
Speed & $5 \mathrm{~km} / \mathrm{hr}$ \\
Tasks & 10 \\
Available Time & $1440 \mathrm{hrs}$ \\
\hline Travel time & $112.9,108.2,111.7 \mathrm{hrs}$ \\
Traveled distances (team 1, team 2, team 3) & $614.5,541,558.7 \mathrm{hrs}$ \\
Utilization & $0.839,0.795,0.791$ \\
Cost & $69,460.18 \mathrm{AED} \dagger$ \\
Total Time of the Done Tasks by all & 821.75 \\
Total Consumed Time including travel time & 1164.59 \\
Team 1, Team2, Team 3 total slack time in 60 days & $77.1,98.3,100.01 \mathrm{hrs}$ \\
\hline$\dagger$ Cost=cost of travel +cost of teams + cost of extra tasks + cost of short tasks &
\end{tabular}

$$
=(1519.7 \times 10 / 5)+([16+15+14] \times 60 \times 8)+(0)+(7000 \times 6 / 60+10000 \times 2 / 6)=69,460.18 \text { AED. }
$$


Finally, a more advanced instance is considered to demonstrate the robustness of the model. The instance is a slightly modified replicate from a real maintenance provider in UAE. Here, 37 different maintenance tasks are considered that are associated with utilities services. The tasks are to be scheduled on a weekly basis with a repetitive pattern, hence, a planning period of one week is enough for a complete schedule. The average travelling speed is set to $40 \mathrm{~km} / \mathrm{hr}$. The remaining data is shown in Tables 8 and 9 .

Table 8

Data of 37 tasks. All costs are in AED.

\begin{tabular}{|c|c|c|c|c|c|c|c|c|c|}
\hline Task no & $d_{m}(\mathrm{hr})$ & $f_{m}$ & $C_{m}^{s p}$ & $C_{m}^{s h}$ & Task no & $d_{m}(\mathrm{hr})$ & $f_{m}$ & $C_{m}^{s p}$ & $C_{m}^{s h}$ \\
\hline 1 & 0 & 7 & 0 & 0 & 20 & 1.4 & 5 & 600 & 6490 \\
\hline 2 & 0 & 7 & 0 & 0 & 21 & 0.7 & 6 & 1800 & 5200 \\
\hline 3 & 0.4 & 7 & 300 & 8270 & 22 & 1.2 & 7 & 900 & 12140 \\
\hline 4 & 0.9 & 4 & 1000 & 3680 & 23 & 0.5 & 4 & 600 & 800 \\
\hline 5 & 1.2 & 2 & 700 & 16800 & 24 & 1.7 & 7 & 700 & 14990 \\
\hline 6 & 0.7 & 3 & 100 & 8250 & 25 & 0.8 & 4 & 1900 & 15510 \\
\hline 7 & 0.5 & 5 & 600 & 3540 & 26 & 0.5 & 5 & 100 & 430 \\
\hline 8 & 0.5 & 6 & 600 & 13910 & 27 & 0.3 & 5 & 1500 & 8730 \\
\hline 9 & 0.5 & 7 & 800 & 15380 & 28 & 0.7 & 5 & 300 & 11240 \\
\hline 10 & 0.4 & 2 & 900 & 7360 & 29 & 0.4 & 4 & 600 & 8690 \\
\hline 11 & 1.6 & 6 & 900 & 3730 & 30 & 4 & 7 & 0 & 16540 \\
\hline 12 & 0.6 & 5 & 2000 & 19320 & 31 & 0.3 & 5 & 1700 & 1760 \\
\hline 13 & 0.6 & 6 & 300 & 9660 & 32 & 0.7 & 2 & 1200 & 3950 \\
\hline 14 & 3 & 6 & 500 & 15230 & 33 & 1.2 & 3 & 1300 & 17840 \\
\hline 15 & 0.8 & 4 & 1800 & 13520 & 34 & 3 & 5 & 1000 & 1300 \\
\hline 16 & 0.4 & 3 & 1600 & 3140 & 35 & 0.2 & 7 & 1600 & 19450 \\
\hline 17 & 0.5 & 3 & 1500 & 10470 & 36 & 1.8 & 4 & 1400 & 50 \\
\hline 18 & 1.3 & 6 & 1700 & 15870 & 37 & 0.7 & 3 & 600 & 6990 \\
\hline 19 & 0.6 & 3 & 600 & 18030 & & & & & \\
\hline$C_{w}=$ & 20 & & & & & & & & \\
\hline
\end{tabular}

Table 9

Distances of 37 tasks in $\mathrm{km}$.

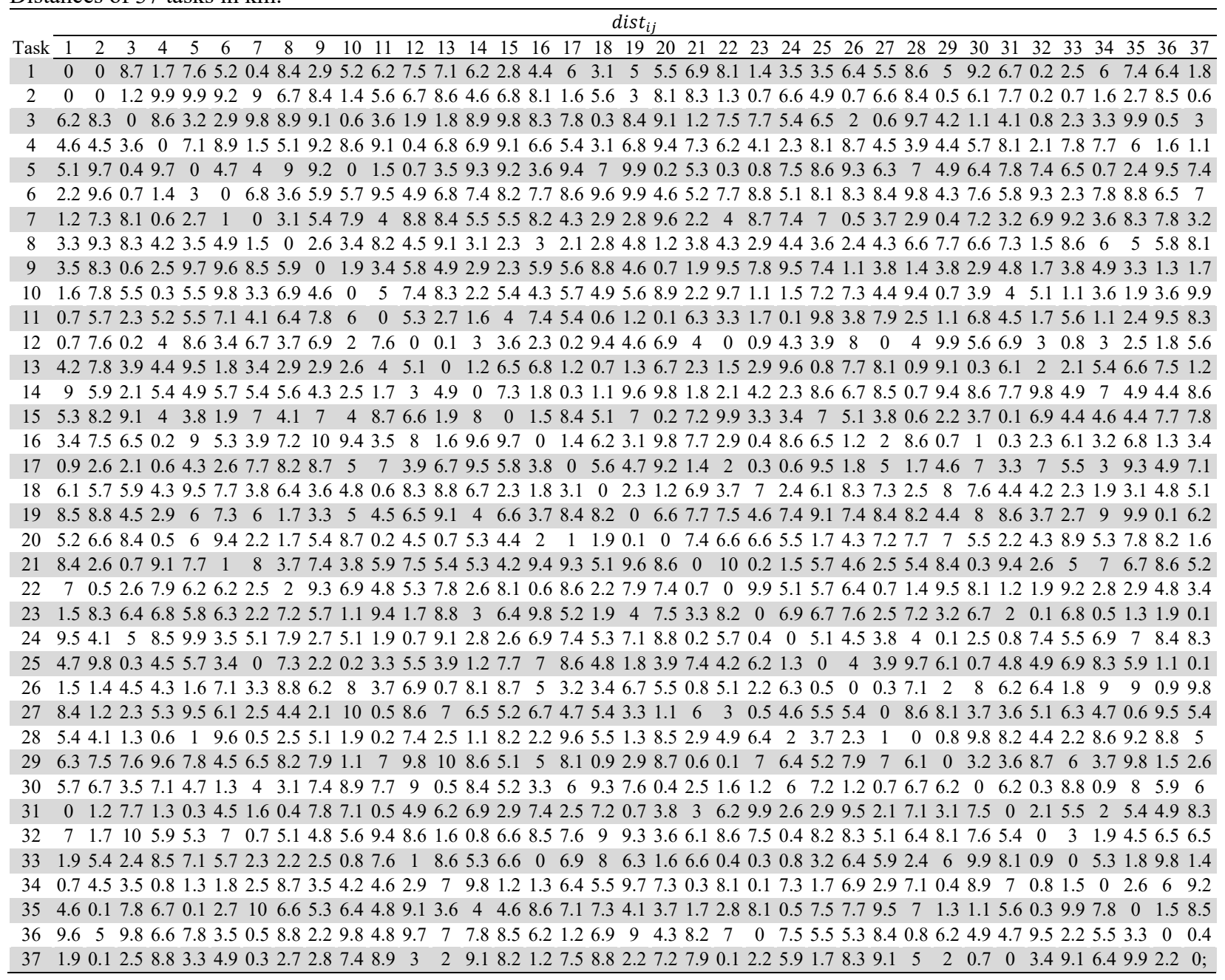


First, we show the optimal results and then compare it by another scenario in which we enforce the hiring of only 3 teams. Note that by fixing the number of teams to 3 , the teams cost will remain constant in the objective function, however, the routing, shortage and excess costs are still optimized. Table 10 shows that not only the optimal solution presents a perfect service level of no shortage, it also demonstrates a lower cost. Although the travelled distance by three teams option will be less than that travelled by the four teams, with only three teams, 7 tasks will go short resulting in a huge penalty that causes a dramatic increase in the cost. While the average team utilization is almost $100 \%$ when three teams are employed, it is less in the optimal solution. In fact, the total time that can be put by only three teams is $168 \mathrm{hrs}$ while the minimum possible length of an unconstrained schedule is $176.4 \mathrm{hrs}$. Therefore, even if the utilization is high, a shortage will remain in the schedule. However, for the case of 4 teams, the total time that can be put by the teams is $224 \mathrm{hrs}$. A slack time will always result due to the resulting time voids at the end of each day that cannot be filled by short tasks. Although higher efficiency and utilization are observed in the case of 3 teams which is intuitively due to higher work requirements as comparted to the case of 4 teams, however, this solution does not result in minimum costs.

Table 10

Results of a schedule of 37 tasks

\begin{tabular}{|c|c|c|}
\hline Parameters/Outputs & Optimal solution & Enforced 3 teams solution \\
\hline Rate & $1 \mathrm{AED} / \mathrm{hr}$ & 1AED/hr \\
\hline Speed & $40 \mathrm{~km} / \mathrm{hr}$ & $40 \mathrm{~km} / \mathrm{hr}$ \\
\hline Tasks & 37 & 37 \\
\hline Available Time of all teams & $224 \mathrm{hrs}$ & $168 \mathrm{hrs}$ \\
\hline Minimum required time of tasks without constraints (back-to-back) & 176.4hrs & $176.4 \mathrm{hrs}$ \\
\hline Travel time & $20.490 \mathrm{hrs}$ & $15.002 \mathrm{hrs}$ \\
\hline Traveled distances (team 1 , team 2 , team 3 ) & $819.600 \mathrm{~km}$ & $600.100 \mathrm{~km}$ \\
\hline Cost of travel time & 20.490AED & 15.002AED \\
\hline Efficiency & $78.7 \%$ & $87.6 \%$ \\
\hline Utilization & $87.9 \%$ & $96.5 \%$ \\
\hline Short tasks & 0 & 12 tasks: $(3,11,13,14,30,34,36)$ \\
\hline Extra tasks & 0 & 0 \\
\hline Cost of short tasks & 0 & 89200AED \\
\hline Cost of excess tasks & 0 & 0 \\
\hline Cost of hired teams alone & 4480.000 & 3360.000 \\
\hline Total Cost & 5000.490 & 92575.002AED \\
\hline Total Time of the Done Tasks by all & $176.4 \mathrm{hrs}$ & $147.2 \mathrm{hrs}$ \\
\hline Total consumed time including travel time & $196.89 \mathrm{hrs}$ & $162.202 \mathrm{hrs}$ \\
\hline Slack time & Team 1: $2.3525 \mathrm{hrs}$ & Team 1: $2.795 \mathrm{hrs}$ \\
\hline
\end{tabular}

\section{Managerial Implications}

Like any other optimization approach, some coefficients/parameters have to be readily available to solve the proposed model. While the parameters like the task times, distances, costs, feasible routes, sites connectivity and team availability are assumed to be on hand and deterministic in their behavior, this is not always the case in real applications. Such parameters may vary over time according to the maintenance scenarios. Breakdowns, interruptions and emergency repairs can cause disturbance that restricts the smooth operation of the proposed schedules in this study. Moreover, having multi-skilled teams entails additional expenses and higher wages. From the monitoring point of view, as multitaskers can perform all types of tasks at any time, the supervision and control of the teams in terms of communication and updates need to be considered with a high capability team manager.

\section{Conclusion}

In this article, a maintenance scheduling model is proposed which aims to reduce the cost of maintenance teams, spare parts, travel time and noncompliance to the service levels via proposing schedules that demonstrate the shortest routes between task sites subject to capacity and time restrictions. The model incorporates a novel formulation of the routing constraints and travel times between tasks sites. Here, a Mixed Integer Linear Programming (MILP) was used to formulate the model. In this formulation, various joint decisions are considered which include selecting the components to maintain, identifying the number of teams to perform the maintenance, specifying the minimal routes and finally establishing a time schedule for performing the tasks.

Various scenarios for maintenance assignments were considered involving different numbers of tasks and teams over different planning periods. In all scenarios, different travel speeds have been tested. While task durations and nominal frequencies have an impact on the resulting schedules, the task sites and travel speeds demonstrated an impact on task sequence on different days. Lower speeds not only increase the cost of travel, but also lead to longer travel times, thereby consuming the available maintenance time and resulting in a difference between the efficiencies and utilizations. Not only the utilization that gets affected by lower speed, the number of hired teams also increases for the same reason. 
Finally, the proposed model helps maintenance providers establish schedules that reduce costs under realistic time, distance, and team availability constraints. In particular, the proposed model can be applied by companies, which provide maintenance services to geographically distributed systems and infrastructure such as commercial buildings and malls.

\section{References}

Bressi, S., Santos, J., \& Losa, M. (2021). Optimization of maintenance strategies for railway track-bed considering probabilistic degradation models and different reliability levels. Reliability Engineering \& System Safety, $207,107359$.

British Standards Institution, BS3811 (1984). Glossary of maintenance terms in Terotechnology, BSI, London.

Camci, F. (2015) Maintenance scheduling of geographically distributed assets with prognostics information. European Journal of Operational Research, 245(2), 506-516.

Chen, Y., Cowling, P., Polack, F., Remde, S., \& Mourdjis, P. (2017) Dynamic optimisation of preventative and corrective maintenance schedules for a large scale urban drainage system. European Journal of Operational Research, 257(2), 494510 .

Cho, D.I., \& Parlar, M., (1991). A survey of maintenance models for multi-unit systems. European Journal of Operational Research, 51(1), 1-23.

Dekker, R. (1996). Applications of maintenance optimization models: a review and analysis. Reliability Engineering \& System safety, 51(3), 229-240.

Dhillon, B.S., (2002). Engineering maintenance: a modern approach. cRc press.

Doostparast, M., Kolahan, F., \& Doostparast, M. (2014) A reliability-based approach to optimize preventive maintenance scheduling for coherent systems. Reliability Engineering \& System, 126, 98-106.

Fontecha, J.E., Guaje, O.O., Duque, D., Akhavan-Tabatabaei, R., Rodríguez, J.P., \& Medaglia, A.L., (2020). Combined maintenance and routing optimization for large-scale sewage cleaning. Annals of Operations Research, 286(1), 441-474.

Fan, D., Ren, Y., Feng, Q., Zhu, B., Liu, Y., \& Wang, Z., (2019). A hybrid heuristic optimization of maintenance routing and scheduling for offshore wind farms. Journal of Loss Prevention in the Process Industries, 62, 103949.

Golpîra, H., \& Tirkolaee, E.B., (2019). Stable maintenance tasks scheduling: A bi-objective robust optimization model. Computers \& Industrial Engineering, 137, 106007.

Hedjazi, D., Layachi, F., \& Boubiche, D.E., (2019). A multi-agent system for distributed maintenance scheduling. Computers \& Electrical Engineering, 77, 1-11.

Irawan, C.A., Ouelhadj, D., Jones, D., Stålhane, M., \& Sperstad, I.B. (2017) Optimisation of maintenance routing and scheduling for offshore wind farms. European Journal of Operational, 256(1).

Mathlouthi, I., Gendreau, M., \& Potvin, J.Y. (2018). Mixed integer linear programming for a multi-attribute technician routing and scheduling problem. INFOR: Information Systems and Operational Research, 56(1), 33-49.

Patwardhan A., Verma A.K., \& Kumar U. (2016). A Survey on Predictive Maintenance Through Big Data. In: Kumar U., Ahmadi A., Verma A., Varde P. (eds) Current Trends in Reliability, Availability, Maintainability and Safety. Lecture Notes in Mechanical Engineering. Springer, Cham. https://doi.org/10.1007/978-3-319-23597-4 31

Rashidnejad, M., Ebrahimnejad, S., \& Safari, J. (2018). A bi-objective model of preventive maintenance planning in distributed systems considering vehicle routing problem. Computers \& Industrial Engineering, 120, 360-381.

Rice, W. F., Cassady, C. R., \& Nachlas, J. A. (1998, May). Optimal maintenance plans under limited maintenance time. In Proceedings of the seventh industrial engineering research conference (pp. 1-3).

Suen, S.L., \& Sen, L. (2004). Mobility options for seniors. Transportation in an ageing society: A Decade of Experience, Proceedings, 27, 97-113.

Yang, L., Zhao, Y., \& Ma, X. (2019). Group maintenance scheduling for two-component systems with failure interaction. Applied Mathematical Modelling, 71, 118-137.

Yu, A.J., \& Seif, J., (2016). Minimizing tardiness and maintenance costs in flow shop scheduling by a lower- bound-based GA. Computers \& Industrial Engineering, 97, 26-40.

Zamorano, E., \& Stolletz, R., (2017). Branch-and-price approaches for the multiperiod technician routing and scheduling problem. European Journal of Operational Research, 257(1), 55-68.

Zhong, S., Pantelous, A.A., Goh, M., \& Zhou, J. (2019). A reliability-and-cost-based fuzzy approach to optimize preventive maintenance scheduling for offshore wind farms. Mechanical Systems and Signal Processing, 124, 643-663. 
(C) 2022 by the authors; licensee Growing Science, Canada. This is an open access article distributed under the terms and conditions of the Creative Commons Attribution (CCBY) license (http://creativecommons.org/licenses/by/4.0/). 